Revista Electrónica Complutense de Investigación en Educación Musical ISSNe: 1698-7454

https://dx.doi.org/10.5209/reciem.60768

\title{
La tecnología digital en la Educación Musical: una revisión de la literatura científica
}

\author{
Diego Calderón-Garrido ${ }^{1}$; Pablo Cisneros²; Isaac Diego García ${ }^{3}$; Rosa de las Heras-Fernández ${ }^{4}$
}

Recibido: 27 de junio de 2018 / Aceptado: 21 de enero de 2019

Resumen. La Educación Musical se caracteriza por una transformación constante en la cual muchos docentes se han sumado a las diferentes propuestas que ofrece la tecnología digital en el proceso de enseñanza-aprendizaje y el acercamiento a nuevas posibilidades y nuevo alumnado, respondiendo además a las necesidades de éste. El presente trabajo realiza una revisión descriptiva de la literatura científica más relevante de los últimos diez años centrada en el uso de la Tecnología Educativa. Dicha revisión, es esencial ya que se trata de una línea educativa en constante evolución y cambio debido a los avances de a la tecnología digital. Los resultados muestran cómo todos los ciclos educativos han sido investigados. Así pues, en el caso de Educación Infantil se detecta el diseño de software específico; en Primaria se ha observado que el alumnado con ciertos conocimientos previos de música ha desarrollado significativamente sus competencias; en Secundaria se muestra una transversalidad de aprendizajes a pesar de que se detecta cierto uso para reforzar contenidos tradicionales desaprovechando las nuevas posibilidades, también se refleja el uso que el docente hace de las tecnologías de la información y la comunicación (TIC) en su formación continua; en Educación Superior destaca la docencia online como nuevo modelo de negocio educativo y un intento constante de incluir las experiencias de fuera del aula dentro de ésta; en las enseñanzas habilitantes llama la atención el uso del portafolios como herramienta evaluativa, formativa y una superación de la unidireccionalidad de las primeras propuestas; y, por último, en los ámbitos educativos con necesidades especiales se hace un mayor hincapié en el desarrollo de competencias interpersonales e intrapersonales.

Palabras clave: Educación Musical; Educación Artística; Tecnología Educativa; tecnologías digital; tecnologías de la información y la comunicación; reforma educativa; situaciones de aprendizaje.

\section{[en] Digital technology in Music Education: a review of the literature}

\begin{abstract}
Music education is characterized by a constant transformation in which many teachers have joined the different proposals offered by digital technology in the teaching-learning process and the approach to new possibilities and new students, also responding to the needs of this. This paper makes a descriptive review of the most relevant scientific literature of the last ten years focused on the use of educational technology. This revision is essential because is an educational topic in constant evolution and change due to advances related to digital technology. The results show how all the educational cycles have been investigated. Thus, in the case of early childhood education, the design of specific software is
\end{abstract}

1 Universidad Internacional de La Rioja (España)

E-mail: diego.calderon@unir.net

2 Universidad Internacional de La Rioja (España)

E-mail: pablo.cisneros@unir.net

3 Universidad Internacional de La Rioja (España)

E-mail: isaacdiego.garcia@unir.net

4 Universidad Internacional de La Rioja (España)

E-mail: rosa.heras@unir.net 
detected; In primary school it has been detected that students with certain previous knowledge of music have significantly developed their skills; In secondary school, there is a transversality of learning, despite the fact that some use is detected to reinforce traditional content, wasting the new possibilities, the teacher's use of information and communication technologies (ICT) in their continuing education is also shown; in higher education, online teaching stands out as a new educational business model and a constant attempt to include experiences from outside the classroom within it; in professional education, emphasis has been placed on the use of portfolios as a formative evaluative tool, and in overcoming the unidirectionality of the first proposals; and in educational settings with special needs, greater emphasis is placed on the development of interpersonal and intrapersonal skills.

Keywords: Music Education; Art Education; Educational Technology; digital technology; information and communication technologies; education reform; learning situations.

Sumario. 1. Introducción. 2 Objetivo. 3. El uso de la tecnología en la Educación Musical. 3.1. Educación Infantil y Primaria. 3.2. Educación Secundaria. 3.3. Educación Superior. 3.4. Formación habilitante. 3.5. Ámbitos con necesidades educativas especiales. 4. Conclusiones. 5. Referencias bibliográficas.

Cómo citar: Calderón-Garrido, D., Cisneros, P., García, I. D., Fernández, D. y de las HerasFernández, R. (2019). La tecnología digital en la Educación Musical: una revisión de la literatura científica. Revista Electró-nica Complutense de Investigación en Educación Musical, 16, 43-55.

\section{Introducción}

La Educación Musical a través de la Historia, ha estado caracterizada por la enseñanza presencial en la que un maestro transmitía sus conocimientos de una forma casi mística a sus discípulos, creándose un vínculo de acompañante y acompañado a través del camino del conocimiento (Gustems-Carnicer, Calderón-Garrido y CalderónGarrido, 2014). Si bien esto era comprensible y loable en épocas pasadas, en pleno siglo XXI puede resultar, si no obsoleto, sí falto de ideas que permitan aprovechar los recursos que los avances tecnológicos ofrecen.

En este sentido, multitud de docentes siguen usando unas metodologías que se podrían denominar tradicionales, centrándose en el desarrollo artesanal de las competencias y saberes. Sin embargo, cada vez son más las propuestas y las voces que claman por readaptar los diversos currículos e incluir las tecnologías digitales en los procesos musicales de enseñanza-aprendizaje musicales (Soutcott y Crawford, 2011). Para ello, además de un conocimiento de las posibilidades y herramientas que el docente tiene a su alcance, es necesario un esfuerzo por incorporar dichas herramientas en las actividades cotidianas del aula (Román, 2017).

Estos esfuerzos responden al alumnado de hoy, que reclama ventanas mediáticas a través de las cuales pueda aprender, crear e imaginar nuevos contextos (Kim, 2016). Un alumnado que está conectado al mundo a través de sus cada vez más sofisticados teléfonos móviles (Bromley, 2012; Kongaut y Bohlin, 2016) y tablets que son usadas como generadoras, grabadoras, gestoras y editoras de sonido (Stephenson y Limbrick, 2015). Un alumnado, en definitiva, alfabetizado en los entornos digitales (Drew, 2012; Hagood y Skinner, 2012) y multimodales (Gainer, 2012) que demanda una educación en este ámbito.

De esta forma, el rol del docente de música se transforma y pasa a ser el de facilitador, acercando al discente al mundo de fuera del aula, a través de esas ventanas que la tecnología permite abrir (Cremata y Powel, 2017). Para ello, por ejemplo, el uso de la web 2.0 ha permitido diseñar proyectos educativos en los que las escuelas más alejadas puedan beneficiarse de la Educación Musical (Crawford, 2013), como es el 
caso de Music X, un proyecto basado en el aprendizaje semipresencial (b-learning), en Australia (Crawford, 2017).

\section{Objetivo}

El objetivo de este artículo es hacer una revisión de la literatura científica más reciente, centrada en las investigaciones que tienen como protagonista el uso de la tecnología y los medios digitales en la Educación Musical. De esta forma, se actualiza y reafirma el conocimiento que se tiene sobre este ámbito y sus aplicaciones en el aula.

\section{El uso de la tecnología en la Educación Musical}

A pesar de que, obviamente, no en todos los países el sistema educativo y la legislación emplea la misma terminología para los diversos ciclos formativos, edad de los discentes y grados referidos a la música y la educación general (Cox y Stevens, 2017), para poder analizar el uso de la Tecnología Educativa en la Educación Musical, en este artículo se emplea la distribución de las diferentes etapas educativas que marca la legislación española.

\subsection{Educación Infantil y Primaria}

En el caso de la Educación Infantil, se han diseñado diversos software como es el caso del SAMI (Software para el aprendizaje de la música en Educación Infantil). Se trata de un proyecto basado en la tecnología móvil que ha obtenido excelentes resultados (PauleRuiz, Álvarez-García, Pérez-Pérez, Álvarez-Sierra y Trespalacios-Menéndez, 2017).

Por su parte, Huang y Yeh (2014) usaron un interface gráfico para desarrollar las habilidades compositivas del alumnado. Los resultados mostraron altos niveles de satisfacción con el aprendizaje realizado y una actitud positiva hacia la actividad musical.

En el caso de Educación Primaria, tanto docentes como discentes han sido estudiados. En el primer caso, según Perkmen y Cevik (2010), los maestros que se ven a sí mismos como sociables, organizados y cuidadosos están más motivados en el uso de la enseñanza asistida por ordenador.

Respecto al alumnado de Primaria, si bien a priori puede parecer una edad prematura para desarrollar el pensamiento computacional, Sáez-Lopez y Sevillano-García (2017) realizaron un experimento con un grupo de 109 estudiantes en el que implementó una serie de actividades basadas en la programación y otro grupo de control de 35 alumnos que no desarrollaron tal tarea. Los resultados mostraron en el grupo experimental un mayor incremento del interés hacia la música, a la vez que trabajaron la programación y desarrollaron dicha competencia de una forma destacada.

Conclusiones parecidas resaltó Hayes (2017) tras implementar su proyecto Sound, Electronics and Music con 900 estudiantes escoceses. Además, los estudios muestran cómo el alumnado desarrolla sus habilidades mediadas por la tecnología con un alto componente reflexivo en su uso (Addesi, Anelli, Benghi y Friberg, 2017).

Destacan estudios como el de Hernández-Bravo, Cardona-Molto y HernándezBravo (2016), quienes evaluaron el efecto de un programa de Educación Musical individualizado en 90 estudiantes de Primaria. Las conclusiones mostraron que los 
estudiantes con un nivel musical medio o alto desarrollaron significativamente la expresión vocal e instrumental, la competencia auditiva, el lenguaje musical, el movimiento y la danza, así como otras competencias artísticas afines. Sin embargo, los estudiantes con un nivel musical bajo no mostraron beneficios significativos en comparación con un aprendizaje tradicional.

A conclusiones parecidas llegaron Shibazaki y Marshall (2013) con su estudio con discentes de 10 y 11 años en Inglaterra. En sus investigaciones, además, demostraron diferencias en función del género a la hora de seleccionar criterios compositivos en el uso de las tecnologías digitales a diferencia de la metodología tradicional. Los resultados reflejaron que los niños se sintieron más atraídos que las niñas por el uso, pero que son éstas las que integraron mejor los conocimientos previos.

En cualquier caso, todos los software diseñados para esta etapa destacan por mantener, al igual que en la metodología tradicional, el espíritu lúdico (Gower y McDowall, 2012).

\subsection{Educación Secundaria}

En la etapa de Educación Secundaria, la literatura científica refleja numerosas investigaciones que ponen de manifiesto el uso de múltiples recursos relacionados con las tecnologías digitales por parte de los docentes. Dicho uso no se limita al aula y al alumnado, sino que también guía la formación continua y el desarrollo profesional de los docentes (Rodesiler, 2015; Savage, 2010).

Por ejemplo, Wise, Greenwood y Davis (2011) describieron las prácticas y percepciones de nueve profesores en Nueva Zelanda y de cómo su metodología se caracteriza por el uso de dispositivos móviles. Este uso u otros parecidos, implican cambios metodológicos en los que el docente se abre ante las nuevas posibilidades y a la creatividad aparejada a las mismas (Wise, 2016).

Por lo que se refiere al alumnado, Lorenzo-Quiles, Vilchez-Fernández y HerreraTorres (2015) realizaron un programa con un grupo de control y otro experimental que usó objetos de aprendizaje digital para música. Los resultados confirmaron que el grupo experimental adquirió un mayor logro académico disciplinar.

Por su parte, Chan, Jones, Scalon y Joiner (2006) usaron el CD-ROM comercial Teach me Piano Deluxe en Inglaterra. En su experimento 36 estudiantes mejoraron significativamente sus habilidades lectoras y rítmicas, involucrando un desarrollo en otras áreas de aprendizaje que implican la redacción, el desarrollo auditivo, la autoconfianza o el nivel de comprensión.

Pero no solo las herramientas específicamente musicales tienen cabida. Por ejemplo, Countriman y Rose (2017) analizaron los beneficios del uso de un videojuego online en el aula de música. Con su experiencia, los autores, además de describir el desarrollo de las competencias musicales y en especial de escucha, reflexionaron sobre los beneficios en el bienestar del alumnado al llevar a la escuela algo que les es tan cercano como los videojuegos. Así pues, y tal como ya se ha sugerido en otras áreas, la integración del día a día del alumnado en el aula ofrece múltiples beneficios, tal como afirman Gertrudix y Gertrudix tras su revisión bibliográfica (2014). A las mismas conclusiones llegaron Pinhati y Siqueira (2014) al considerar superlativa la importancia de la realidad social de cada estudiante a la hora de diseñar las actividades musicales. En este sentido, la educación formal, la no formal y la informal se dan la mano en el aula (Stowell y Dixon, 2014). En esta línea, tal como propone Carlisle 
(2011), se puede pensar incluso en la creación de nuevas experiencias interpretativas en las que tomen protagonismo la música que escuchan los adolescentes, proveniente en muchos casos de los diversos medios de comunicación y las redes sociales que se usan en el siglo XXI, convirtiéndose así en experiencias multimodales donde la audición y la creación se dan la mano.

Sin embargo, estudios realizados por Savage (2010) en el Reino Unido mostraron que el uso de las TIC se emplea en la mayoría de los casos para reforzar contenidos tradicionales, limitando, e incluso malgastando, así sus posibilidades. Otro handicap que se ha reflejado con asiduidad es la falta de recursos en los centros, en especial los que dependen directamente de los diferentes estados (Crawford, 2009). Este dato, además, se complementa con el análisis de diversos currículos educativos que no reflejan el reconocimiento social que existe sobre el uso de la tecnología en los procesos de enseñanza-aprendizaje (Crawford y Southcott, 2017; Peppler, 2010; Liu y Liu, 2017).

\subsection{Educación Superior}

En Educación Superior, las tecnologías digitales han supuesto un revulsivo y una revolución en el modelo de negocio del sector de la educación, surgiendo los mercados de educación online (OEM), proveedores de educación online (OEP) y servicios de educación en línea (OES) (Pathak, 2016). Obviamente, la Educación Musical que se imparte en estos ámbitos debe su existencia y su razón de ser a las TIC, por lo que no es necesario incidir aún más en sus beneficios.

En el caso de las enseñanzas presenciales, las investigaciones muestran múltiples bondades en el uso de determinadas herramientas. Así, por ejemplo, King (2008) reflejó que el uso del LTI (Learning Technology Interface) en el ámbito de la percusión desarrollaba una planificación más eficaz en el conjunto de tareas y que, además, cuanto más usaron los alumnos el LTI el desarrollo pasó a ser exponencial. En este sentido, la multimodalidad y la hipertextualidad característica de los formatos digitales ha resultado catalizadora en la rápida aceptación por parte de discentes y docentes (Domingo, 2012; Edwards, Perry, Janzen y Menzies, 2012). Esta misma multimodalidad está resultando clave a la hora de poder comunicar al resto del mundo los avances en la investigación musical (Yang, Ketner, Luker y Patterson, 2016). A su vez, está sirviendo como soporte para poder fomentar un trabajo transversal que integre diversas áreas de conocimiento (Boche y Henning, 2015).

Si algo caracteriza a la sociedad actual es la conexión constante con el resto del mundo. Esta ventana abierta a través de los teléfonos móviles supone un revulsivo en algunos contextos que, si bien pueden parecer a simple vista contrarios a la concentración necesaria para asimilar un aprendizaje, bien aprovechados y gestionados pueden ser tremendamente beneficiosos. A tal conclusión llegó Niu (2017) con su investigación basada en la creación de una plataforma accesible a través de los dispositivos móviles.

Llama la atención el estudio que Pike (2017) realizó con docentes de música en su formación inicial. En su investigación exploró tanto al docente como al discente y las actitudes de ambos en la relación que se establecía en un entorno online. Las conclusiones mostraban una rápida adaptación de los futuros maestros hacia esta modalidad y una mejor comprensión de los contenidos por parte del alumnado. Por otro lado, Talski (2015) realizó una serie de actividades online con 161 futuros do- 
centes. Su conclusión fue que la motivación hacia el aprendizaje era mayor que en el caso de la enseñanza presencial. Igualmente, Vidulin-Orbanić y Duraković (2011) afirmaron que los futuros docentes encontraban en las tecnologías digitales una manera de optimizar su tiempo y llegar al alumnado más directamente en comparación con la transmisión clásica de conocimientos musicales.

Estudios parecidos con futuros maestros muestran cómo el uso de podcats, que buscan trabajar la unión de las historias personales con la formación e inquietudes del alumnado, afianzan la autoestima y refuerzan la personalidad, a la vez que desarrollan la creatividad de los docentes (Bolden y Nahachewsky, 2015).

\subsection{Formación habilitante}

En el ámbito de la formación instrumental especializada, la enseñanza online es la que más adeptos ha ganado. En esta modalidad se han estudiado tanto los comportamientos del profesorado como del alumnado. Dye (2016) examinó el uso de video conferencias, analizando los diferentes patrones de comportamiento de 6 docentes y discentes estadounidenses. Las conclusiones mostraban un alto grado de aprovechamiento de las clases y una comunicación bidireccional.

Esto, a su vez, demuestra cómo la unidireccionalidad de las primeras acciones está siendo superada para plantearse nuevas propuestas en las que la retroalimentación y la comunicación fluida son el objetivo (Kão y Niitsoo, 2014). Para ello, algunos software incluyen, como parte de sus posibilidades, aplicaciones de interacción entre docente y discente e, incluso, entre los propios discentes, creándose así comunidades de aprendizaje (Waldron, 2013).

Sin embargo, no solo la enseñanza online ha sido desarrollada. En ámbitos tan especializados como la formación instrumental, las tecnologías digitales han mostrado sorprendentes resultados al combinar metodologías convencionales con los nuevos modelos. Tal es el caso de lo reflejado por Roy (2016) en entornos tan, a priori, poco favorecedores como la transmisión oral de la música tradicional de la India. En estos ámbitos, las herramientas sincrónicas y asincrónicas ofrecen posibilidades que, hasta ahora, eran impensables, como, por ejemplo, componer música en grupo desde diferentes partes del mundo (Biasutti, 2015; Dobson y Littleton, 2016; Naughton, 2012), o la creación de foros virtuales de reflexión (Merga, 2014). A su vez, trabajar con la tecnología en la etapa formativa ha abierto el camino profesional de los jóvenes intérpretes que se han sentido atraídos, entre otras cosas, por la ingeniería de sonido o la acústica (Kardos, 2012).

En la formación instrumental, Nijs y Leman (2014) mostraron los beneficios del uso del sistema interactivo Music Paint Machine durante nueve meses, comparando un grupo experimental con uno de control de estudiantes de clarinete. Los investigadores afirmaron que los resultados en el grupo experimental fueron sorprendentemente mejores que en el grupo de control en lo referido a competencias instrumentales.

Por su parte, Rowe, Triantafyllaki y Anagnotopoulou (2015), tras trabajar con 19 estudiantes de piano durante seis semanas el sistema de improvisación Miror, concluyeron que la capacidad de improvisación del alumnado había mejorado notablemente, en especial si comparaban las capacidades adquiridas, con los avances que se solían conseguir en las clases tradicionales.

No cabe duda de que en un aprendizaje que requiere de tanto seguimiento continuo -y que, además, trabaja con un material tan etéreo como el sonido--, una he- 
rramienta que permita reflejar todos los avances como un portafolios digital es un recurso de primer orden (Taylor, Dunbar-Hall y Rowley, 2012). En esta línea trabajaron Brook y Upitis (2015), quienes en sus investigaciones con el e-portfolio iSCORE concluyeron, además, que éste promueve la autorregulación de los estudiantes.

Al hilo de lo anterior, y en la recopilación del producto sonoro, Silveira y Gavin (2016) realizaron varios experimentos basados en la grabación, descomposición y posterior autoevaluación de 112 estudiantes de música. Su análisis mostró una gran profundidad en las reflexiones previas a la autoevaluación y un seguimiento de todos los parámetros de la música que sin las tecnologías necesarias serían indescifrables. En este sentido, el procesamiento del sonido y descomposición de todos los elementos que conforman la música permiten a los estudiantes centrarse y optimizar esfuerzos en mejorar partes o elementos concretos de su interpretación y su técnica, ya sea la afinación, la exactitud rítmica, etc., sin verse interferidos por otros aspectos (Cano, Schuller y Dittmar, 2014). Incluso las nuevas posibilidades que se abren han permitido centrarse en el gesto musical, tal como relataron Van der Linden, Schoonderwaldt, Bird y Johnson (2011) al referirse a la observación, análisis y posterior corrección de la inclinación del arco del violín.

En cualquier caso, algunos estudios muestran que son los menores de 25 años, los hombres y las personas con mayor nivel educativo los que se sienten más atraídos hacia el uso de las tecnologías digitales en su formación musical, ya sea inicial o de reciclaje (Suki, 2011).

En un nivel profesional, las tecnologías digitales suponen un gran avance y un replanteamiento de lo que hasta ahora eran los tradicionales conciertos pedagógicos y la recepción de la música (Lepa, Hoklas, Egermann y Weinzieri, 2015). Con esto, se puede afirmar que se cierra el círculo. Es decir, el que, hasta hace poco tiempo era estudiante, se convierte en un profesional que dedica parte de su tiempo a enseñar desde el escenario a jóvenes estudiantes. En esta función, las tecnologías digitales tendrán un papel protagonista.

\section{5. Ámbitos con necesidades educativas especiales}

A pesar de que la literatura científica no ha desarrollado en demasía esta línea, los ámbitos con necesidades educativas especiales merecen, sin duda, una especial atención. Sin embargo, el estudio llevado a cabo por Cano y Sánchez-Iborra (2015) con alumnado con dificultades de aprendizaje en el que se usaba la herramienta multimedia PLAIME (Platform for the Ingration of handicapped children in Music Education) mostraba un avance sustancial en sus conocimientos musicales y un desarrollo positivo del comportamiento.

Por su parte, Hillier, Greher, Queenan, Marshall y Kopec (2016) realizaron un proyecto con personas con trastorno del espectro autista durante nueve semanas. A lo largo de este tiempo aplicaron el programa SoundScape. Obtuvieron excelentes beneficios relacionados con la disminución del estrés y la ansiedad.

En contextos hospitalarios, la tecnología digital ha permitido, en cierta manera y a través de intervenciones personalizadas, normalizar la vida de los pacientes. En este sentido, Issaka y Hopkins (2017) recogen las conclusiones de un experimento llevado a cabo en Australia.

Los últimos avances han mostrado sus bondades incluso en alumnado con deficiencias auditivas, tal como destaca la investigación de Chao-Fernández, Román- 
García y Chao-Fernández (2017). En ésta, se relata una experiencia basada en la historia en línea que concluye reconociendo el incremento en la motivación del alumnado y la integración facilitada por los contextos virtuales.

\section{Conclusiones}

La literatura científica expone cómo, en general, la tecnología digital presenta grandes beneficios tanto en docentes como en discentes. Así pues, en el caso de los primeros, los estudios reflejan la creación de redes profesionales y foros de debate. Igualmente, el uso de las TIC ayuda en la formación continua del profesorado. Sin embargo, a pesar de que se han registrado múltiples cambios metodológicos, algunos estudios han mostrado un desaprovechamiento de la tecnología digital por parte del profesorado, limitándose a un refuerzo de los contenidos tradicionales. Otro de los aspectos negativos que se ha observado es la falta de recursos por parte de las instituciones.

Respecto al alumnado, se muestran un gran desarrollo de las competencias interpersonales e intrapersonales. Así pues, los estudios muestran cómo el uso de las TIC en el aula promueve la sociabilidad, la motivación, el espíritu crítico, la reflexión, la autoconfianza, la autoestima y la autorregulación, disminuyendo el estrés y algunos estados de ansiedad. Igualmente, las TIC favorecen el desarrollo de competencias musicales, tanto instrumentales como vocales, reforzándose así el dominio del lenguaje musical, la audición y la expresión corporal. De la misma forma, la literatura refleja el desarrollo en áreas de conocimiento afines, así como del pensamiento computacional. Se han registrado también experiencias muy positivas en procesos de autoevaluación al poder descomponer el sonido en todos sus parámetros, de forma que el alumnado pueda así hacer hincapié en cada uno de los aspectos, e incluso mejorar la actitud muscular a través de las grabaciones audiovisuales. Destacan las propuestas de creaciones de portafolios digitales en los cuales el alumnado pueda incluir las grabaciones de sus interpretaciones. En cualquier caso, los estudios muestran cómo son los varones con mayor nivel de estudios los que se sienten más atraídos por las TIC, a pesar de que son las mujeres las que mejor las integran en su vida cotidiana.

Respecto al proceso enseñanza-aprendizaje, las TIC han fomentado la multimodalidad e hipertextualidad fusionando diversos lenguajes e incluyendo las redes sociales en el proceso. Destaca también la facilidad que tiene la tecnología digital para unir los ámbitos de fuera con los de dentro del aula. En este sentido, se han registrado experiencias que animan a integrar el teléfono móvil en el proceso educativo. Es destacable también el desarrollo de las plataformas digitales y entornos online que permiten optimizar el tiempo y llegar a un mayor número de alumnado. Así, al mejorarse la tecnología, se han solventado muchos de los problemas relativos a la transmisión unidireccional característica de años atrás.

De esta forma, desde la más temprana edad hasta los ámbitos universitarios, pasando por los centros habilitantes, tales como conservatorios y escuelas de música, los investigadores han narrado empírica y epistemológicamente las bondades en diversos contextos, culturas y usos de la tecnología. Se abren así nuevos caminos en los que también se involucran nuevos públicos, alumnado futuro, que sin la tecnología mediante, no podría disfrutar de una educación musical y los beneficios que conlleva. En este artículo se ha recopilado y mostrado tanto recursos como el efecto que éstos tienen en sus usuarios. Ya solo queda soñar con un futuro en el que los 
docentes pierdan el miedo a estas tecnologías. Un futuro en el que profesorado y alumnado vivan la misma realidad.

\section{Referencias bibliográficas}

Addessi, A. R., Anelli, F., Benghi, D., y Friberg, A. (2017). Child-Computer Interaction at the Beginner Stage of Music Learning: Effects of Reflexive Interaction on Children's Musical Improvisation. Frontiers in psychology, 8. https://doi.org/10.3389/fpsyg.2017.00065

Biasutti, M. (2015). Creativity in virtual spaces: Communication modes employed during collaborative online music composition. Thinking skills and creativity, 17, 117-129. https://doi.org/10.1016/j.tsc.2015.06.002

Boche, B., y Henning, M. (2015). Multimodal Scaffolding in the Secondary English Classroom Curriculum. Journal of adolescent \& adult literacy, 58(7), 579-590. https:// doi.org/10.1002/jaal.406

Bolden, B., y Nahachewsky, J. (2015). Podcast creation as transformative music engagement. Music Education Research, 17(1), 17-33. https://doi.org/10.1080/14613808.2014.969219

Brook, J., y Upitis, R. (2015). Can an online tool support contemporary independent music teaching and learning? Music education research, 17(1), 34-47. https://doi.org/10.1080/ 14613808.2014.969217

Bromley, K. (2012). Using Smartphones to Supplement Classroom Reading. Reading Teacher, 66(4), 340-344. https://doi.org/10.1002/TRTR.01130

Cano, E., Schuller, G., y Dittmar, C. (2014). Pitch-informed solo and accompaniment separation towards its use in music education applications. EURASIP Journal on Advances in Signal Processing, 2014(1), 23. https://doi.org/10.1186/1687-6180-2014-23

Cano, M. D., y Sánchez-Iborra, R. (2015). On the use of a multimedia platform for music education with handicapped children: A case study. Computers \& Education, 87, 254276. https://doi.org/10.1016/j.compedu.2015.07.010

Carlisle, K. (2011). Conceptualising secondary aurality and its impact on possibility for engagement of children and adolescents within school music settings. Music Education Research, 13(2), 241-253. https://doi.org/10.1080/14613808.2011.577766

Chao-Fernández, R., Román-García, S., y Chao-Fernández, A. (2017). Online Interactive Storytelling as a strategy for learning music and for integrating pupils with hearing disorders into Early Childhood Education (ECE). Procedia-Social and Behavioral Sciences, 237, 17-22. https://doi.org/10.1016/j.sbspro.2017.02.005

Chan, L. M. Y., Jones, A. C., Scanlon, E., y Joiner, R. (2006). The use of ICT to support the development of practical music skills though acquiring keyboard skills: a classroom based study. Computers \& Education, 46(4), 391-406. https://doi.org/10.1016/j. compedu.2004.08.007

Countryman, J., y Rose, L. S. (2017). Wellbeing In The Secondary Music Classroom: Ideas From Hero'S Journeys And Online Gaming. Philosophy of Music Education Review, 25(2), 128-149. http://www.jstor.org/stable/10.2979/philmusieducrevi.25.2.03

Cox, G., y Stevens, R. (2017). The origins and foundations of music education. International perspectives. Londres: Bloomsbury. https://doi.org/10.14742/ajet.268

Crawford, R. (2009). Secondary school music education: A case study in adapting to ICT resource limitations. Australasian Journal of Educational Technology, 25(4), 471-488. https://doi.org/10.14742/ajet.1124 
Crawford, R. (2013). Evolving technologies require educational policy change: Music education form the 21st century. Australasian Journal of Educational Technology, 29(5), 717-734. https://doi.org/10.14742/ajet.268

Crawford, R. (2017). Rethinking teaching and learning pedagogy for education in the twenty first century: blended learning in music education. Music Education Research, 19(2), 195-213. http://dx.doi.org/10.1080/14613808.2016.1202223

Crawford, R., y Southcott, J. (2017). Curriculum stasis the disconnect between music and technology in the Australian curriculum. Technology Pedagogy and Education, 26(3), 347-366. http://dx.doi.org/10.1080/1475939X.2016.1247747

Cremata, R., y Powell, B. (2017). Online music collaboration project: Digitally mediated, deterritorialized music education. International Journal of Music Education, 35(2), 302315. https://doi.org/10.1177/0255761415620225

Dobson, E., y Littleton, K. (2016). Digital technologies and the mediation of undergraduate students' collaborative music compositional practices. Learning, Media and Technology, 41(2), 330-350. https://doi.org/10.1080/17439884.2015.1047850

Domingo, M. (2012). Linguistic layering: social language development in the context of multimodal design and digital technologies. Learning Media and Technology, 37(2), 177 197. https://doi.org/10.1080/17439884.2012.670645

Drew, S. V. (2012). Open Up the Ceiling on the Common Core State Standards: Preparing Students for 21st-Century LiteracyNow. Journal of adolescent \& adult literacy, 56(4), 321-330. https://doi.org/10.1002/JAAL.00145

Dye, K. (2016). Student and instructor behaviors in online music lessons: An exploratory study. International Journal of Music Education, 34(2), 161-170. https://doi. org/10.1177/0255761415584290

Edwards, M., Perry, B., Janzen, K., y Menzies, C. (2012). Using the Artistic Pedagogical Technology of Photovoice to Promote Interaction in the Online Post-Secondary Classroom: The Students' Perspective. Electronic Journal of E-learning, 10(1), 32-43.

Gainer, J. (2012). Critical Thinking: Foundational for Digital Literacies and Democracy. Journal of adolescent \& adult literacy, 56(1), 14-17. https://eric.ed.gov/?id=EJ969434

Gertrudix Barrio, F., y Gertrudix Barrio, M. (2014). Tools and resources for music creation and consumption on web 2.1. applications and educational possibilities. Educacion XX1, 17(2), 313-336. https://doi.org/10.5944/educxx1.17.2.11493

Gower, L., y McDowall, J. (2012). Interactive music video games and children's musical development. British Journal of Music Education, 29(1), 91-105. https://doi.org/10.1017/ S0265051711000398

Gustems-Carnicer, J., Calderón-Garrido, D., y Calderón-Garrido, C. (2014). Alquimia y pedagogía: Confluencia y paralelismo de una vieja tradición. Encuentros Multidisciplinares, 48, 1-6. http://hdl.handle.net/10486/678656

Hagood, M. C., y Skinner, E. N. (2012), Appreciating Plurality Through Conversations Among Literacy Stakeholders. Journal of adolescent \& adult literacy, 56(1), 4-6. https:// ila.onlinelibrary.wiley.com/doi/pdf/10.1002/JAAL.00093

Hayes, L. (2017). Sound, Electronics, and Music: A Radical and Hopeful Experiment in Early Music Education. Computer Music Journal, 41(3), 36-49. https://doi.org/10.1162/ comj_a_00428

Hernández-Bravo, J. R., Cardona-Molto, MC., y Hernández-Bravo, JA. (2016). The effects of an individualised ICT based music education programme on primary school students' musical competence and grades. Music Education Research, 18(2), 176-195. https://doi. org/10.1080/14613808.2015.1049255 
Hillier, A., Greher, G., Queenan, A., Marshall, S., y Kopec, J. (2016). Music, technology and adolescents with autism spectrum disorders: The effectiveness of the touch screen interface. Music Education Research, 18(3), 269-282. https://doi.org/10.1080/14613808.2015.1077802

Huang, C. F., y Yeh, Y. S. (2014). Graphical interface-based automated music composition use among elementary school students. Musicae Scientiae, 18(1), 84-97. https://doi. org/10.1177/1029864913514596

Issaka, A., y Hopkins, L. (2017). Engagement with education: Music education in a paediatric hospital. International Journal of Educational Research, 83, 142-153. https:// doi.org/10.1016/j.ijer.2017.02.012

Kão, K., \& Niitsoo, M. (2014). MatchMySound: Introducing Feedback to Online Music Education. In Y. Cao, T. Väljataga, J. Tang, H. Leung \& M. Laanpere, New Horizons in Web Based Learning (pp. 217-225). Tallinn: Springer. https://doi.org/10.1007/978-3-31913296-9_24

Kardos, L. (2012). How music technology can make sound and music worlds accessible to student composers in Further Education colleges. British Journal of Music Education, 29(2), 143-151. https://doi.org/10.1017/S0265051712000186

Kim, G. M. (2016). Transcultural Digital Literacies: Cross $\square$ Border Connections and Self $\square$ Representations in an Online Forum. Reading Research Quarterly, 51(2), 199-219. https://doi.org/10.1002/rrq.131

King, A. (2008). Collaborative learning in the music studio. Music Education Research, 10(3), 422-438. https://doi.org/10.1080/14613800802280167

Kongaut, Ch., y Bohlin, E. (2016). Investigating mobile broadband adoption and usage: A case of smartphones in Sweden. Telematics and informatics, 33(3), 742-752. https://doi. org/10.1016/j.tele.2015.12.002

Lepa, S., Hoklas, A-K, Egermann, H., y Weinzieri, S. (2015). Sound, materiality and embodiment challenges for the concept of 'musical expertise' in the age of digital mediatization. Convergence-the international journal or research into new media technologies, 21(3), 294-300. https://doi.org/10.1177/1354856515579837

Liu, Z., y Liu, Y. (2017). Teaching Strategy and Instructional System Construction of Chinese National Instrumental Technology Education. Eurasia Journal of Mathematics, Science and Technology Education, 13(8), 5645-5653. https://doi.org/10.12973/ eurasia.2017.01018a

Lorenzo-Quiles, O., Vilchez-Fernández, N., y Herrera-Torres, L. (2015). Educational effectiveness analysis if the use of digital music learning objects. Comparison of digital versus non-digital teaching resources in compulsory secondary education. Infancia $y$ Aprendizaje, 38(2), 295-326. https://doi.org/10.1080/02103702.2015.1016748

Merga, M. K. (2014). Literacy Agents Online E-Discussion Forums for Advancing Adults' Literacy Practices. Journal of adolescent \& adult literacy, 57(6), 472-482. https://doi. org/10.1002/jaal.271

Naughton, C. (2012). 'Heidegger and Joe:'Revisiting the 'thing'in the context of a student's experience of an online community. British journal of music education, 29(3), 331-341. https://doi.org/10.1017/S0265051712000307

Nijs, L., y Leman, M. (2014). Interactive technologies in the instrumental music classroom: A longitudinal study with the Music Paint Machine. Computers \& Education, 73, 40-59. https://doi.org/10.1016/j.compedu.2013.11.008

Niu, Z. H. (2017). Research on the construction of music teaching cloud platform in colleges and universities for mobile terminal. Agro Food Insdustry Hi-Tech, 28(1), 2774-2778. http://www.teknoscienze.com/tks_issue/vol_281/ 
Pathak, B. K. (2016). Emerging online educational models and the transformation of traditional universities. Electronic Markets, 26(4), 315-321. https://link.springer.com/ article/10.1007/s12525-016-0223-4

Paule-Ruiz, M., Álvarez-García, V., Pérez-Pérez, J. R., Álvarez-Sierra, M., y Trespalacios-Menéndez, F. (2017). Music learning in preschool with mobile devices. Behaviour \& Information Technology, 36(1), 95-111. https://doi.org/10.1080/01449 29X.2016.1198421

Peppler, K. A. (2010). Media Arts: Arts Education for a Digital Age. Teachers Collegue Record, 112(8), 2118-2153. http://kpeppler.com/Docs/2010_Peppler_Media_Arts. pdf

Perkmen, S., y Cevik, B. (2010). Relationship between pre-service music teachers' personality and motivation for computer-assisted instruction. Music Education Research, 12(4), $415-$ 425. https://doi.org/10.1080/14613808.2010.519768

Pike, P. D. (2017). Improving music teaching and learning through online service: A case study of a synchronous online teaching internship. International Journal of Music Education, 35(1), 107-117. https://doi.org/10.1177/0255761415613534

Pinhati, F., y Siqueira, S. W. M. (2014). Music students' behavior on using learning objects closer to the domain characteristics and the social reality. Computers in human behavior, 30, 760-770. https://doi.org/10.1016/j.chb.2013.10.039

Rodesiler, L. (2015). The nature of selected English teachers' online participation. Journal of Adolescent \& Adult Literacy, 59(1), 31-40. https://doi.org/10.1002/jaal.427

Román, M. (2017). Technology at the service of music education. Revista Española de Pedagogía, 75(268), 481-495. https://doi.org/10.22550/REP75-3-2017-17

Rowe, V., Triantafyllaki, A., y Anagnostopoulou, X. (2015). Young pianists exploring improvisation using interactive music technology. International Journal of Music Education, 33(1), 113-130. https://doi.org/10.1177/0255761414540137

Roy, J. (2016). The Internet Guru: Online Pedagogy in Indian Classical Music Traditions. Asian Music, 47(1), 103-135. https://doi.org/10.1353/amu.2016.0001

Sáez-Lopez, J. M. y Sevillano-García, M. L. (2017). Sensors, programing and devices in Art Education sessions. One case in the context of primary education. Cultura y educación, 29(2), 350-384. https://doi.org/10.1080/11356405.2017.1305075

Savage, J. (2010). A survey of ICT usage across English secondary schools. Music Education Research, 12(1), 89-104. https://doi.org/10.1080/14613800903568288

Shibazaki, K., y Marshall, N. A. (2013). Gender differences in computer -and instrumentalbased musical composition. Educational Research, 55(4), 347-360. https://doi.org/10.10 80/00131881.2013.844937

Silveira, J. M., y Gavin, R. (2016). The effects of audio recording and playback on selfassessment among middle school instrumental music students. Psychology of Music, 44(4), 880-892. https://doi.org/10.1177/0305735615596375

Southcott, J., y Crawford, R. (2011). The intersections of curriculum development: Music, ICT and Australian music Education. Australasian Journal of Educational Technology, 27(1), 122-136. https://doi.org/10.14742/ajet.987

Stephenson, J., y Limbrick, L. (2015). A Review of the Use of Touch-Screen Mobile Devices by People with Developmental Disabilities. Journal of autism and developmental disorders, 45(12), 3777-3791. https://doi.org/10.1007/s10803-013-1878-8

Stowell, D., y Dixon, S. (2014). Integration of informal music technologies in secondary school music lessons. British Journal of Music Education, 31(1), 19-39. https://doi. org/10.1017/S026505171300020X 
Suki, N. M. (2011). Gender, Age, and Education: Do They Really Moderate Online Music Acceptance?. Communications of the IBIMA, 2011, 1-18. https://doi. org/10.5171/2011.959384

Talsik, E. (2015). The investigation of readiness for e-learning of pre-service music teachers in Turkey. Anthropologist, 21(1-2), 263-270. https://doi.org/10.1080/09720073.2015.11 891815

Taylor, J., Dunbar-Hall, P., y Rowley, J. (2012). The e-portfolio continuum: Discovering variables for e-portfolio adoption within music education. Ascilite, 28(2), 1362-1381. https://doi.org/10.14742/ajet.776

Van Der Linden, J., Schoonderwaldt, E., Bird, J., y Johnson, R. (2011). Musicjacketcombining motion capture and vibrotactile feedback to teach violin bowing. IEEE Transactions on Instrumentation and Measurement, 60(1), 104-113. https://doi. org/10.1109/TIM.2010.2065770

Vidulin-Orbanić, S., y Duraković, L. (2011). The influence of educational technology on the development of music students'competences. Hrvatski časopis za odgoj i obrazovanje, 13(2), 124-160. https://hrcak.srce.hr/76348

Waldron, J. (2013). YouTube, fanvids, forums, vlogs and blogs: Informal music learning in a convergent on-and offline music community. International Journal of Music Education, 31(1), 91-105. https://doi.org/10.1177/0255761411434861

Wise, S., Greenwood, y J., Davis, N. (2011). Teachers' use of digital technology in secondary music education: illustrations of changing classrooms. British Journal of Music Education, 28(2), 117-134. https://doi.org/10.1017/S0265051711000039

Wise, S. (2016). Secondary school teachers' approaches to teaching composition using digital technology. British Journal of Music Education, 33(2), 283-295. https://doi.org/10.1017/ S0265051716000309

Yang, L., Ketner, K., Luker, S., y Patterson, M. (2016). A complete system for publishing music- related ETDs Technology development and publishing model. Library hi tech, 34(1), 151-163. https://doi.org/10.1108/LHT-10-2015-0096 